\title{
Multimodality advanced imaging and intervention in gland diseases
}

Dedicated imaging of specific body areas and anatomical structures, such as glands, requires integrated knowledge of different advanced methods (1-3). Furthermore, even for many of the gland pathologies, interventional radiology allows obtaining surgical results using advanced and minimally invasive techniques (4-6).

Given this continuous evolution of diagnostic and interventional imaging, the purpose of this special issue, entitled "Multimodality advanced imaging and intervention in gland diseases", is to give an overview of the most recent and relevant topics on diagnostic and interventional imaging in glands.

The first article deals with the imaging study of pancreatic pathologies, which represents, in some cases, a diagnostic challenge, also considering the importance of instrumental diagnosis as an indication for surgical treatment (7-10). The contribution of Bicci et al., "Pancreatic neuroendocrine tumors", provides a comprehensive review of the imaging features and diagnostic approach to pancreatic neuroendocrine neoplasms. The article by Vacca et al. "MR severity index assessed by T1 weighted imaging for acute pancreatitis: correlation with clinical outcomes and grading of the revised Atlanta Classification" focuses instead on the value of imaging in the staging of inflammatory pathology of the pancreas using MRI $(11,12)$.

In recent years, dual-energy CT technology has been one of the main innovations in sectional imaging diagnostics, with application first in the musculoskeletal and neuroradiological field, but with potential applications for various abdominal organs and pathologies (13-18). In their article "New advances in CT imaging of pancreas diseases", Agostini et al. review this and other advanced CT imaging possibilities in pancreas disorders. Another interesting article dealing with the applications of dual-energy CT is the contribution by Gentili et al., "Dual-energy CT in gland tumors: a comprehensive review and differential diagnosis", that covers the various advantages and clinical benefits of the use of this advanced technique in the imaging of gland neoplasms $(19,20)$.

Breast imaging plays a fundamental role in clinical surveillance, staging, and follow-up of both benign and malignant pathology (21-24). In addition to mammography and ultrasound, magnetic resonance imaging is the fundamental second-level diagnostic modality, thanks to the possibility of obtaining functional information through the use of contrast medium and specific MR sequences $(25,26)$. De Cataldo et al. illustrate in their article "Apparent diffusion coefficient MRI (ADC-MRI) in axillary breast cancer lymph node metastases detection" the role of magnetic resonance imaging in the study of pathological lymph nodes.

The use of contrast-enhanced imaging and diffusion-weighted MR sequences are also the main features of the multiparametric MRI of the prostate (27-29). Palumbo et al., in their contribution "Biparametric and multiparametric MRI approach to prostate cancer disease: a long-standing debate on dynamic contrast enhancement", address the current and discussed issue of the diagnostic approach to prostate cancer through the use of biparametric and multiparametric MRI, reviewing the possible advantages and limitations that emerged from the literature. The multiparametric study of the prostate, together with recent developments in fusion-imaging technology, are the basis for the evolving changes in the approach to prostate biopsy; this focus is the topic of the article by Ziglioli et al., "Multiparametric MRI in the management of prostate cancer: an update. A narrative review" (30,31).

Among gland diseases, salivary gland pathologies benefit from the multiparametric imaging study, mainly thanks to advanced ultrasonography techniques $(32,33)$. The two contributions of this Issue, "Ultra-high Frequency Ultrasound (UHFU) applications in Sjogren syndrome: current concepts" by Aringhieri et al., and "Multiparametric ultrasound in parotid gland evaluation" by Martino et al., provide a comprehensive overview of the actual applications and available technology for the advanced US imaging of the salivary glands (34-36).

In abdominal imaging, the pathology of the adrenal glands poses important differential diagnoses for which radiology plays a fundamental role in providing valuable clinical information (37,38); Reginelli et al. highlight the role of multimodality imaging in adrenal lesions, along with the possible pitfalls, in their article "Pitfalls and differential diagnosis on adrenal lesions: current concepts in CT/MR imaging" (39-41).

The last contribution of the volume is the work of Tortora et al., "Pituitary Magnetic Resonance Imaging vs. Bilateral 
Inferior Petrosal Sinus Sampling: comparison between non-invasive and invasive diagnostic techniques for Cushing's Disease"; this interesting and focused topic illustrates the value of the global approach of imaging modalities, with both the diagnostic and interventional point of view (42-44).

With the articles selected in this volume, thanks to the great efforts and expertise of the Authors, we have tried to give the radiologist's point of view on the most recent innovations in the field of diagnostic and interventional imaging of gland diseases, in an era in which the integration of clinical knowledge and the multidisciplinary approach represents a fundamental strategy for patient management.

A renewed heartfelt thanks to all the authors, with the hope of having made a valuable contribution to all readers.

\section{Acknowledgments}

Funding: None.

\section{Footnote}

Provenance and Peer Review: This article was commissioned by the editorial office, Gland Surgery for the series "Multimodality Advanced Imaging and Intervention in Gland Diseases”. The article did not undergo external peer review.

Conflicts of Interest: The author has completed the ICMJE uniform disclosure form (available at http://dx.doi.org/10.21037/ gs-20-592). The series "Multimodality Advanced Imaging and Intervention in Gland Diseases" was commissioned by the editorial office without any funding or sponsorship. AB serves as an unpaid editorial board member of Gland Surgery from Jun 2018 to May 2020 and served as the unpaid Guest Editor of the series.

Ethical Statement: The author is accountable for all aspects of the work in ensuring that questions related to the accuracy or integrity of any part of the work are appropriately investigated and resolved.

Open Access Statement: This is an Open Access article distributed in accordance with the Creative Commons AttributionNonCommercial-NoDerivs 4.0 International License (CC BY-NC-ND 4.0), which permits the non-commercial replication and distribution of the article with the strict proviso that no changes or edits are made and the original work is properly cited (including links to both the formal publication through the relevant DOI and the license). See: https://creativecommons.org/licenses/by-nc-nd/4.0/.

\section{References}

1. Bruno F, Arrigoni F, Mariani S, et al. Advanced magnetic resonance imaging (MRI) of soft tissue tumors: techniques and applications. Radiol Med 2019;124:243-52.

2. Gentili F, Pelini V, Lucii G, et al. Update in diagnostic imaging of the thymus and anterior mediastinal masses. Gland Surg 2019;8:S188-207.

3. Jacobs MA, Ouwerkerk R, Wolff AC, et al. Monitoring of neoadjuvant chemotherapy using multiparametric, (2)(3)Na sodium MR, and multimodality (PET/CT/MRI) imaging in locally advanced breast cancer. Breast Cancer Res Treat 2011;128:119-26.

4. Barile A, Quarchioni S, Bruno F, et al. Interventional radiology of the thyroid gland: critical review and state of the art. Gland Surg 2018;7:132-46.

5. Ierardi AM, Carrafiello G. Diagnostic and interventional radiology in emergency setting and glands. Gland Surg 2019;8:107.

6. Carrafiello G. Interventional radiology of glands. Gland Surg 2018;7:57-8.

7. Ierardi AM, Biondetti P, Coppola A, et al. Percutaneous microwave thermosphere ablation of pancreatic tumours. Gland Surg 2018;7:59-66.

8. Liu Y, Wang Y, Tang W, et al. Multiparametric MR imaging detects therapy efficacy of radioactive seeds brachytherapy in pancreatic ductal adenocarcinoma xenografts. Radiol Med 2018;123:481-8.

9. Biondetti P, Fumarola EM, Ierardi AM, et al. Bleeding complications after pancreatic surgery: interventional radiology 
management. Gland Surg 2019;8:150-63.

10. Angileri SA, Gorga G, Tortora S, et al. Biliary injuries after pancreatic surgery: interventional radiology management. Gland Surg 2019;8:141-9.

11. Maldonado I, Shetty A, Estay MC, et al. Acute Pancreatitis Imaging in MDCT: State of the Art of Usual and Unusual Local Complications. 2012 Atlanta Classification Revisited. Curr Probl Diagn Radiol 2020;S0363-0188(20)30057-8.

12. Expert Panel on Gastrointestinal Imaging, Porter KK, Zaheer A, et al. ACR Appropriateness Criteria(R) Acute Pancreatitis. J Am Coll Radiol 2019;16:S316-30.

13. Rizzo S, Femia M, Radice D, et al. Evaluation of deep myometrial invasion in endometrial cancer patients: is dual-energy CT an option? Radiol Med 2018;123:13-9.

14. Travieso-Aja MDM, Maldonado-Saluzzi D, Naranjo-Santana P, et al. Diagnostic performance of contrast-enhanced dual-energy spectral mammography (CESM): a retrospective study involving 644 breast lesions. Radiol Med 2019;124:1006-17.

15. Foti G, Beltramello A, Minerva G, et al. Identification of residual-recurrent cholesteatoma in operated ears: diagnostic accuracy of dual-energy CT and MRI. Radiol Med 2019;124:478-86.

16. Carotti M, Salaffi F, Beci G, et al. The application of dual-energy computed tomography in the diagnosis of musculoskeletal disorders: a review of current concepts and applications. Radiol Med 2019;124:1175-83.

17. Agostini A, Borgheresi A, Mari A, et al. Dual-energy CT: theoretical principles and clinical applications. Radiol Med 2019;124:1281-95.

18. Agliata G, Schicchi N, Agostini A, et al. Radiation exposure related to cardiovascular CT examination: comparison between conventional 64-MDCT and third-generation dual-source MDCT. Radiol Med 2019;124:753-61.

19. Schieda N, Siegelman ES. Update on CT and MRI of Adrenal Nodules. AJR Am J Roentgenol 2017;208:1206-17.

20. Chawla A, Srinivasan S, Lim TC, et al. Dual-energy CT applications in salivary gland lesions. Br J Radiol 2017;90:20160859.

21. Yoshida T, Urikura A, Shirata K, et al. Short tau inversion recovery in breast diffusion-weighted imaging: signal-to-noise ratio and apparent diffusion coefficients using a breast phantom in comparison with spectral attenuated inversion recovery. Radiol Med 2018;123:296-304.

22. Barker SJ, Anderson E, Mullen R. Magnetic resonance imaging for invasive lobular carcinoma: is it worth it? Gland Surg 2019;8:237-41.

23. Brzezinska M, Dixon JM. Inflammatory breast cancer: no longer an absolute contraindication for breast conservation surgery following good response to neoadjuvant therapy. Gland Surg 2018;7:520-4.

24. Ashikari AY, Kelemen PR, Tastan B, et al. Nipple sparing mastectomy techniques: a literature review and an inframammary technique. Gland Surg 2018;7:273-87.

25. Alzaghal AA, DiPiro PJ. Applications of Advanced Breast Imaging Modalities. Curr Oncol Rep 2018;20:57.

26. van Nijnatten TJA, Ploumen EH, Schipper RJ, et al. Routine use of standard breast MRI compared to axillary ultrasound for differentiating between no, limited and advanced axillary nodal disease in newly diagnosed breast cancer patients. Eur J Radiol 2016;85:2288-94.

27. Cutaia G, Tosto G, Cannella R, et al. Prevalence and clinical significance of incidental findings on multiparametric prostate MRI. Radiol Med 2020;125:204-13.

28. Beyhan M, Sade R, Koc E, et al. The evaluation of prostate lesions with IVIM DWI and MR perfusion parameters at 3T MRI. Radiol Med 2019;124:87-93.

29. Abdollahi H, Mofid B, Shiri I, et al. Machine learning-based radiomic models to predict intensity-modulated radiation therapy response, Gleason score and stage in prostate cancer. Radiol Med 2019;124:555-67.

30. Zawaideh JP, Sala E, Pantelidou M, et al. Comparison of Likert and PI-RADS version 2 MRI scoring systems for the detection of clinically significant prostate cancer. Br J Radiol 2020;93:20200298.

31. Bae JH, Kim SH. Transrectal ultrasound-guided prostate biopsy versus combined magnetic resonance imaging-ultrasound fusion and systematic biopsy for prostate cancer detection in routine clinical practice. Ultrasonography 2020;39:137-43.

32. Vogl TJ, Albrecht MH, Nour-Eldin NA, et al. Assessment of salivary gland tumors using MRI and CT: impact of experience on diagnostic accuracy. Radiol Med 2018;123:105-16.

33. Kim DW. Computed tomography features of the major salivary glands after radioactive iodine ablation in patients with papillary thyroid carcinoma. Radiol Med 2018;123:20-7.

34. Cindil E, Oktar SO, Akkan K, et al. Ultrasound elastography in assessment of salivary glands involvement in primary Sjogren's 
syndrome. Clin Imaging 2018;50:229-34.

35. Bhatia KSS, Dai YL. Routine and Advanced Ultrasound of Major Salivary Glands. Neuroimaging Clin N Am 2018;28:273-93.

36. Onkar PM, Ratnaparkhi C, Mitra K. High-frequency ultrasound in parotid gland disease. Ultrasound Q 2013;29:313-21.

37. Hanafy AK, Mujtaba B, Roman-Colon AM, et al. Imaging features of adrenal gland masses in the pediatric population. Abdom Radiol (NY) 2020;45:964-81.

38. Warda MH, Shehata SM, Zaiton F. Chemical-shift MRI versus washout CT for characterizing adrenal incidentalomas. Clin Imaging 2016;40:780-7.

39. Foti G, Malleo G, Faccioli N, et al. Characterization of adrenal lesions using MDCT wash-out parameters: diagnostic accuracy of several combinations of intermediate and delayed phases. Radiol Med 2018;123:833-40.

40. Lanza C, Galeazzi V, Carboni N, et al. Neuroblastoma image-defined risk factors in adrenal neuroblastoma: role of radiologist. Gland Surg 2019;8:S168-77.

41. d'Amuri FV, Maestroni U, Pagnini F, et al. Magnetic resonance imaging of adrenal gland: state of the art. Gland Surg 2019;8:S223-32.

42. Varrassi M, Cobianchi Bellisari F, Bruno F, et al. High-resolution magnetic resonance imaging at $3 \mathrm{~T}$ of pituitary gland: advantages and pitfalls. Gland Surg 2019;8:S208-15.

43. Raveendranath V, Nagarajan K, Umamageswari A, et al. Three-dimensional magnetic resonance-based morphometry of pituitary stalk. Radiol Med 2019;124:206-10.

44. Radvany MG, Quinones-Hinojosa A, Gallia GL, et al. Venous Sampling for Cushing Disease: Comparison of Internal Jugular Vein and Inferior Petrosal Sinus Sampling. Endocr Pract 2016;22:1057-61.

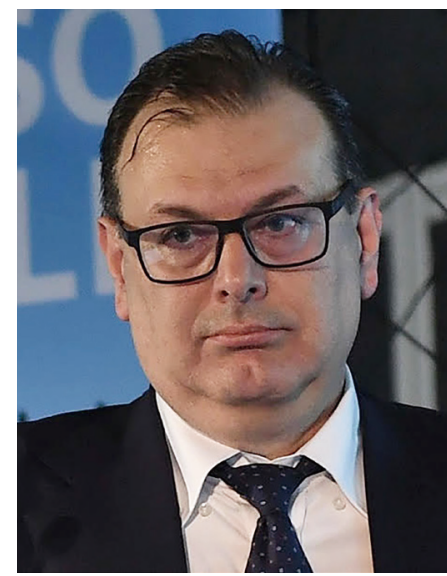

Antonio Barile

Antonio Barile, MD

Department of Biotechnology and Applied Clinical Sciences, University of L'Aquila, L'Aquila, Italy.

(Email: antonio.barile@univaq.it)

Submitted Jul 02, 2020. Accepted for publication Jul 24, 2020.

doi: $10.21037 / \mathrm{gs}-20-592$

View this article at: http://dx.doi.org/10.21037/gs-20-592

Cite this article as: Barile A. Multimodality advanced imaging and intervention in gland diseases. Gland Surg 2020;9(6):22112214. doi: $10.21037 /$ gs-20-592 